\title{
Cancer Cell Growth - A Mini Review Part-1: Proliferation, Nutrient, Warburg Effect
}

\section{Koyunoglu C*}

Energy Institute, Istanbul Technical University, Istanbul, 34469, Turkey

\begin{abstract}
The objective of this work is to address the mechanism how cancer cell growth. Subsequently, the mechanisms that control the cells progress through the cell cycle are very maintained with evolution. Throughout metastatic development, distributing cancer cells end up being lodged within the microvasculature of end organs, where most pass away from mechanical contortion. Although this phenomenon was very first explained over a half-century earlier, the systems making it possible for specific cells to endure this metastasis-suppressive obstacle stay unidentified. In order to hold up against the extreme ambient of cancer cells, strong tumors should likewise enhance source of nourishment usage once sources are limited. In this part of mini review Proliferation, Nutrient and Warburg Effect terms summarized.
\end{abstract}

Keywords: Cancer; Biochemistry; Breast cancers; Glycolysis

\section{Introduction}

Comprehending the cellular basis of cancer indicates having the ability explain the biochemistry of the regulated paths in between cell surface area and nucleus that manage cell development. Two simultaneous current seminar on the activation and restraint of oncogenic expansion offered much new details on the balance of regulative impacts that constrain the development of typical cells and fail in cancer [1].

\section{Methods}

The elements of cell development such as Proliferation, Nutrient, Warburg Effect are summed up gathered from the information of research study works.

\section{Proliferation}

Among the trademarks of cancer is unrestrained cell proliferation. In many, if not all, human tumors, several of the cell-cycle checkpoints is jeopardized such that cells multiply in an uncontrolled way. A comprehensive however still insufficient understanding of the systems that collaborate occasions that regulate cell proliferation has actually originated from molecular analyses of human tumors, typical tissues, and animal designs [2].

The proliferation of cancer cells in mice with reduced EMT was considerably enhanced, as revealed formerly in mouse models of breast cancers [3].

Cancer control by adaptive immunity includes a variety of specified and clearance systems. Nevertheless, effective inhibition of rapid cancer development by $\mathrm{T}$ cells and interferon- $\gamma(\mathrm{IFN}-\gamma)$ needs extra undefined systems that apprehend cancer cell proliferation [4].

Here we reveal that reduced activity of ASS1 in cancers supports proliferation by assisting in pyrimidine synthesis by means of CAD (carbamoyl-phosphate synthase 2, aspartate transcarbamylase, and dihydroorotase complex) activation [5].

Nearly every human tumor is accompanied by several anomalies in genes that impact control of cell cycle, resulting in uncontrolled cell proliferation. Therefore, human tumors can be defined in part by the kind of cellcycle control that the tumor cells have actually lost [2].

Nutrient. The majority of cancer cells take in greater quantities of glucose, and produce far more lactate than regular cells, even in the presence of sufficient O2. This phenomenon, called aerobic glycolysis or the Warburg effect, supports tumor cell development [6].

The new model anticipates the presence of 'vital' values of cancer cells' nutrient supply and their capability to take in nutrients. Near these values even a extremely little modification can turn an unrestrained, deadly cancer into a non-growing ('inactive') or passing away ('regressive') tumor, and vice versa [7].

Warburg effect. In the 1920s, Otto Warburg, a German biochemist, showed that unlike typical tissues, cancer cells constantly upregulated glycolysis even when oxygen was plentiful. This phenomenon of socalled aerobic glycolysis became ended up being called the Warburg effect [8-10].

How does the Warburg effect can be found in?

Otto Warburg and coworkers were the very first to report that cancer cells continue to engage in glycolysis and lactate production even when oxygen levels are abundant. This is referred to as the Warburg impact. In spite of comprehensive efforts, nevertheless, Warburg and others might not discover anomalies that hindered oxidative metabolic process in cancer cells. As a result, lots of biologists questioned whether Warburg's observations really showed tumor metabolic process or were rather an artifact of unintended hypoxia occuring throughout his in vitro experiments. Imaging researches explained below assistance the in vivo significance of the Warburg effect [11].

\section{Results and Discussion}

There are some researches show the reason or the result of the related topic above mentioned as ordered.

*Corresponding author: Koyunoglu C, Energy Institute, Istanbul Technical University, Istanbul, 34469, Turkey, Tel: +90(212)2853941; E-mail: ckoyunoglu@ itu.edu.tr

Received: December 10, 2018; Accepted: December 17, 2018; Published December 24, 2018

Citation: Koyunoglu C (2018) Cancer Cell Growth - A Mini Review Part-1: Proliferation, Nutrient, Warburg Effect. Biochem Anal Biochem 7: 371. doi: 10.4172/2161-1009.1000371

Copyright: $\odot 2018$ Koyunoglu C. This is an open-access article distributed under the terms of the Creative Commons Attribution License, which permits unrestricted use, distribution, and reproduction in any medium, provided the original author and source are credited. 
- It was revealed that obstructing copper trafficking causes cellular oxidative stress and lowers levels of cellular ATP. The minimized level of ATP causes activation of the AMP-activated protein kinase that results in decreased lipogenesis. Both results add to the restraint of cancer cell proliferation. The work results develop copper chaperones as new targets for future advancements in anticancer treatments [12].

- Multiplying cancer cells display significantly various metabolic demands to a lot of regular distinguished cells [1].

- In order to support their high rates of proliferation, cancer cells take in extra nutrients and divert those nutrients into macromolecular synthesis paths. Metabolic paths should for that reason be rewired in such a way that balances biosynthetic procedures with enough ATP production to support cell development and survival. As all cancer cells depend on this modification in metabolic process, these altered paths represent appealing healing targets. Nevertheless, due to the fact that regular proliferating cells have the exact same metabolic demands as cancer cells, discovering a restorative window in between multiplying cancer cells and proliferating regular cells stays a significant difficulty in the advancement of effective cancer treatments targeting metabolic paths [13].

- The cells of a tumour are highly affected by its stromal element. Proof recommends that there is an exchange of metabolites in between cancer and stromal cells to offer nutrients. Stromal cells can have an essential function in reactive oxygen species (ROS) metabolic process within the tumour compartment. This might be especially vital in keeping the replicative capacity of cancer stem cells. Although the metabolic demands of cancer stem cells have actually not been examined, early stem-cell advancement includes a metabolic switch to glycolysis that is similar to the Warburg effect in cancer [14].

- Notably, advances in cancer metabolic process were extremely interwoven with those of bioenergetics in general. When the Warburg effect was first explained, hardly any was found out about the crosssignaling between respiration and fermentation; the tricarboxylic acid cycle (TCAC) was first explained just in the 1930s (Krebs and Johnson). Nonetheless, Crabtree recommended currently in 1929 that worsened glycolytic activity hindered respiration, not just in tumors however likewise in other mitotically active tissues. The restraint of oxygen usage by glucose in multiplying cells was then called the Crabtree effect. Years later on, Weinhouse (1972) observed that tumor cells have fetal isoforms of some glycolytic enzymes, recommending this could be responsible for their enhanced glycolytic flux [15].

\section{Conclusion}

There are lots of factors that effects basically cancer cell growth since it has to be simplified by using some simplification models as well.

\section{Acknowledgement}

Thanks my mother who encouraged me.

\section{References}

1. Hunter T (1986) Cancer: Cell growth control mechanisms. Nature 322: 14-16.

2. The Cell Cycle and Cancer, in Medical Cell Biology (2018) Chapter 9, Goodman SR (ed.) Academic Press, San Diego 273-289.

3. Zheng X, Carstens JL, Kim J, Scheible M, Kaye J, et al. (2015) Epithelialto-mesenchymal transition is dispensable for metastasis but induces chemoresistance in pancreatic cancer. Nature 527: 525-530.

4. Braumuller H, Wieder T, Brenner E, Abmann S, Hahn M, et al. (2013) T-helper1-cell cytokines drive cancer into senescence. Nature 494: 361-365.

5. Rabinovich S, Adler L, Yizhak K, Sarver A, Silberman A, et al. (2015) Diversion of aspartate in ASS1-deficient tumors fosters de novo pyrimidine synthesis. Nature 527: 379-383.

6. Dong T, Yan Y, Chai H, Chen S, Xiong X, et al. (2015) Pyruvate kinase M2 affects liver cancer cell behavior through up-regulation of HIF-1 $\alpha$ and $\mathrm{Bcl}-\mathrm{xL}$ in culture. Biomed Pharmacother 69: 277-284.

7. Haw M (2000) Humans wobble but they don't fall down. Nature.

8. Ferreira LM (2010) Cancer metabolism: The Warburg effect today. Exp Mol Pathol 89: 372-380.

9. Diaz-Ruiz R, Rigoulet M, Devin A (2011) The Warburg and Crabtree effects: On the origin of cancer cell energy metabolism and of yeast glucose repression. Biochim Biophys Acta 1807: 568-576.

10. Bensinger SJ, Christofk HR (2012) New aspects of the Warburg effect in cancer cell biology. Semin Cell Dev Biol 23: 352-361.

11. Kaelin WG Jr, Thompson CB (2010) Q\&A: Cancer: Clues from cell metabolism Nature 465: 562-564

12. Wang J, Luo C, Shan C, You Q, Lu J, et al. (2015) Inhibition of human copper trafficking by a small molecule significantly attenuates cancer cell proliferation. Nat Chem 7: 968-979.

13. Vander Heiden MG (2011) Targeting cancer metabolism: a therapeutic window opens. Nat Rev Drug Discov 10: 671-684.

14. Schulze A, Harris AL (2012) How cancer metabolism is tuned for proliferation and vulnerable to disruption. Nature 491: 364-373.

15. Ferreira LMR, Hebrant A, Dumont JE (2012) Metabolic reprogramming of the tumor. Oncogene 31: 3999-4011. 